\title{
ARMARIOS DE PAPEL: EL HETEROSEXISMO EN LOS MANUALES DE ESPAÑOL COMO LENGUA EXTRANJERA
}

\section{Resumen}

El presente trabajo pretende analizar la representación de la sexualidad en los manuales de Español como Lengua Extranjera (ELE) y poner de manifiesto el papel que desempeñan estos en la transmisión y perpetuación (directa o indirectamente) de estereotipos y valores heterosexistas y LGBTfóbicos. Se ha seleccionado un corpus representativo y actual de los métodos de ELE, con el objetivo de describir y examinar el tratamiento de los contenidos lingüísticos. La investigación se complementa con una fundamentación teórica que recoge cuestiones como el valor de la otredad en los documentos curriculares - Marco común europeo de referencia para las lenguas (MCER) y Plan Curricular del Instituto Cervantes (PCIC) -, la importancia de los factores afectivos para el aprendizaje de idiomas y, especialmente, el potencial subversivo de la pedagogía queer.

Palabras clave: Teoría queer, orientación sexual, LGTB, español como lengua extranjera, libros de texto.

\section{Abstract}

Paper closets: heterosexism in the textbooks of Spanish as a foreign language

The aim of this paper is to analyse the representation of sexuality in Spanish as a Foreign Language coursebooks, highlighting the role of books in the perpetuation, both directly and indirectly, of heterosexist and LGBTphobic stereotypes and values. Thus a corpus, consisting of a current selection of Spanish textbooks, has been chosen with the aim of examining the treatment given to linguistic contents. The research posits a theoretical basis which explores the relevance of Otherness in curricular documents - Common European Framework of Reference for Languages (CEFR) and Curricular Plan of the Cervantes Institute (IPPC) -, the importance of affective factors in order to succeed in language learning and, essentially, the subversive potential of queer pedagogy.

Keywords: Queer theory, sexual orientation, LGBT, Spanish as a Foreign Language, coursebooks.

\section{Resumo}

Armários de papel: heterossexismo em manuais de espanhol como língua estrangeira

Este trabalho visa analisar a representação da sexualidade nos manuais de espanhol como língua estrangeira (ELE) e, sobretudo, evidenciar o papel que eles desenvolvem na

Universidad Internacional Menéndez Pelayo, España.

Correo electrónico: msalviarod@gmail.com

Dirección postal: Universidad Internacional Menéndez Pelayo, Av. de los Castros, 42, 39005 Santander, Cantabria, España. 
transmissão e perpetuação (direta ou indiretamente) de estereótipos e valores heterossexistas e LGBTfóbicos. Foi selecionado um corpus representativo e atual dos métodos de espanhol como língua estrangeira, com o objetivo de descrever e examinar o tratamento dos conteúdos linguísticos. A investigação é complementada com uma fundamentação teórica que explora questões como o valor da outredade nos documentos curriculares - Quadro europeu comum de referência para as línguas (QECR) e Plano Curricular do Instituto Cervantes (PCIC) -, a importância dos fatores afetivos para a aprendizagem de idiomas e, especialmente, o potencial subversivo da pedagogia queer.

Palabras clave: Teoria queer, orientação sexual, LGBT, espanhol como língua estrangeira, manuais escolares.

\section{Introducción y objetivos}

En los últimos años se han conseguido importantes logros activistas en la comunidad LGBT (Lesbianas, Gais, Bisexuales, Trans), ha aumentado la visibilidad de la diversidad sexual - en la calle y en los medios -, así como el interés por los estudios de género. No obstante, las sexualidades que divergen de la heterosexualidad continúan fuera de los materiales, del aula y del currículo de Español como Lengua Extranjera (ELE).

Se ha justificado este destierro a través de distintos argumentos: que la sexualidad debe tratarse fuera de la clase, que pertenece a un ámbito privado e íntimo, que su mención perjudica la normalización, o que es un tema ajeno a la enseñanza del idioma. Como respuesta a este panorama, se plantea el siguiente trabajo de investigación, cuyos objetivos son:

- Estudiar el tratamiento de la sexualidad en los manuales de ELE, atendiendo especialmente a las sexualidades subversivas (lesbianas, gais, bisexuales), que aparecen en el corpus escogido.

- Analizar cualitativamente los ítems lingüísticos encontrados en el corpus.

- Visibilizar los discursos heterosexistas ${ }^{1}$ (explícitos e implícitos) y LGBTfóbicos en el corpus.

\footnotetext{
El heterosexismo (acuñado por Gide en 1911) remite a la creencia y presuposición de que todas las personas son heterosexuales, rasgo asumido como normal, natural y deseable, con la consiguiente invisibilización, prejuicio y discriminación hacia el resto de opciones sexoafectivas. La heteronormatividad (Warner 1991) es el régimen sociocultural que refuerza y reproduce esta creencia, normaliza y privilegia ciertas conductas, mientras oprime y marginaliza las identidades e individuos que no las cumplen (del Castillo 2015).
} 


\section{Marco Teórico}

\section{Estado de la cuestión}

El análisis de manuales desde el punto de vista de la orientación sexual es un territorio inexplorado en el ámbito del ELE. Esta situación es paradójica teniendo en cuenta que el español es la segunda lengua extranjera más estudiada en el mundo. Además, la falta de investigaciones en esta área «contribuye al mantenimiento del estatus quo de la heteronormatividad» (Pawelczyk et al. 2014, 61; apud Erlman 2015, 18).

Con todo, pueden establecerse tres bloques de fuentes consultadas para el desarrollo de este proyecto.

En primer lugar, hay que mencionar la pionera labor investigadora de Guijarro Ojeda, enfocada al inglés como lengua extranjera en España. Su producción consta de un amplio abanico de artículos, que abarcan desde las aplicaciones didácticas de la teoría queer en la enseñanza de lenguas (Guijarro Ojeda 2004a, 2006; Guijarro Ojeda y Ruiz-Cecilia 2013), la transmisión de valores de género y sexualidad en los manuales de inglés de primaria y secundaria (Guijarro Ojeda 2005a, 2005b), o la utilización de la otredad como recurso didáctico en el currículo de lenguas extranjeras (Guijarro Ojeda 2004b, 2005c).

En segundo lugar, esta vez en el ámbito de ELE, se hallan escasísimas pero valiosas referencias sobre la incorporación de las sexualidades al aula. Cabe señalar las reflexiones y propuestas didácticas de Mazzaro y Chaves (2010), Lluch (2010) y Mazzaro (2013), todas desde Brasil. Los autores advierten del valor cultural de la sexualidad y hacen hincapié en la necesidad de llevarla al ELE como parte de la formación crítica y axiológica de los/as alumnos/as; eso sí, huyendo de clichés y respetando la diversidad.

Finalmente, ha resultado imprescindible la bibliografía anglosajona sobre la introducción de las identidades LGBT en el aula de idiomas. Se trata de un campo de reflexión pedagógica que nace en los 1990 (Nelson 1993; Summerhawk 1998), y que desde entonces ha ido desarrollándose exponencialmente (El-Metoui 2014; Macdonald 2014). Dentro de este ámbito, existen diversos estudios sobre el heterosexismo de los materiales (Gray 2013; del Castillo 2015; Erlman 2015).

\section{Sexualidades en ELE}

\section{Heterosexismo y currículum oculto}

En un contexto de enseñanza que va menguando paulatinamente el sexismo de los materiales y va enriqueciéndose con representaciones de diversidad cultural, cabe preguntarse aún por la omisión sistemática de las sexualidades no normativas: «¿Dónde están gais y lesbianas en los manuales? No se encuentran en 
ningún sitio. Todavía están en el armario. La gente en los manuales nunca es gay. Los árboles genealógicos y las fotos familiares son rigurosamente heterosexuales» (Thornbury 1999, 15-16, apud Cartwright 2006, 176).

Esto se explica porque la educación, en general, y los materiales, en particular, son heterosexistas y reproducen un régimen de heteronormatividad. Y es que las instituciones educativas son agentes con gran poder ideológico, que ejercen a través del currículo. Así, no solo es importante lo que se menciona (que representa aquello que socialmente se considera importante y válido), sino también es fundamental lo que se obvia (el currículum oculto).

Esta omisión es justificada por algunas personas, que argumentan que la sexualidad forma parte de la esfera privada y no es relevante ni apropiada como contenido de una clase de idiomas. No obstante, como apunta Guijarro Ojeda (2006), la sexualidad está plenamente presente en el aula y, si no se aprecia, es porque se camufla en el discurso hegemónico heterosexual. Tan solo basta con reflexionar sobre todas las referencias que se acumulan en los manuales (por no mencionar las interacciones entre docentes y alumnado ${ }^{2}$ ) sobre la familia, los estados civiles, las relaciones o las celebraciones.

Cabe preguntarse entonces, ¿por qué es relevante hablar de las identidades sexoafectivas no normativas? En primer lugar, los/as docentes deben asumir que entre su alumnado hay estudiantes gais, lesbianas y bisexuales (sin necesidad de saber quiénes), que deben enfrentarse no solo a las dificultades lingüísticas y culturales correspondientes al aprendizaje de una lengua extranjera, sino también a la discriminación de currículos y materiales (Nelson 1993, 148).

Por otro lado, las personas heterosexuales están fuera del armario en cada clase, y su opción sexual emerge sin problemas ni estigmas. En cambio, los/as estudiantes LGBT no tienen las mismas oportunidades para su autoexpresión: «Deshomosexualizo las cosas continuamente. Me quito a mí, mis amigos, mi familia, mi comunidad, mi cultura de la escena» (Nelson 1993, 146).

Independientemente del estatus social y legal de lo LGBT en los países de origen del alumnado, las personas LGBT existen y el alumnado también siente curiosidad sobre el tema. E incluso el alumnado más escéptico puede beneficiarse de la introducción de la diversidad sexoafectiva en el aula, pues se pueden fomentar diálogos y debates que mejoren sus recursos lingüísticos y argumentativos.

En definitiva, como apunta Cartwright $(2006,177)$, las personas no heterosexuales representan una minoría significativa en la sociedad, por lo que su exclusión de los manuales no es solo absurda y anacrónica, sino también ofensiva y perjudicial para el aprendizaje. Estudiantes y toda la comunidad LGBT tienen derecho al reconocimiento, la visibilidad y la representación que se les niega en los manuales.

Como propone El-Metoui (2012), imagine el lector por un momento sus planes para el fin de semana. Y ahora intente hablar de ellos sin mencionar a su pareja, familia, amigos, los lugares en que socializa, las revistas que lee, los programas o las películas que ve, y dónde va a comprar. 


\section{Sexualidades disidentes: de la tolerancia al cuestionamiento}

Desde la didáctica de lenguas - básicamente, en el ámbito del English as a Foreign Language (EFL) o English for Speakers of Other Languages (ESOL) - se han planteado dos maneras de incorporar las disidencias sexoafectivas a la práctica educativa: por un lado, lo que Britzman (1995) denomina pedagogía de la inclusión (que parte de un marco teórico Gay \& Lesbian); por otro lado, las pedagogías del cuestionamiento ${ }^{3}$ (resultado de la influencia de la teoría queer).

El principal objetivo de la pedagogía de la inclusión (Britzman 1995) es convertir las prácticas educativas, las clases y los currículos en más inclusivos para lesbianas, gais y bisexuales, introduciendo imágenes y representaciones auténticas de estos. No obstante, aunque bienintencionada, esta propuesta es insuficiente para Nelson (1999, 376-377). Y es que, como comenta Guijarro Ojeda (2004b, 62), las referencias corren el riesgo de quedar diluidas en anécdotas superficiales y silenciar los verdaderos problemas de la comunidad LGBT.

Asimismo, desde el punto de vista ético, este tipo de acercamiento puede interpretarse como una búsqueda de legitimización y aceptación social. El énfasis en la empatía y tolerancia puede reforzar el estatus de minoría y victimización (Nelson 1999, 377).

La otra propuesta pedagógica, la del cuestionamiento, analiza y cuestiona todo el espectro de identidades sexuales (también la heterosexualidad), y cómo operan sobre ellas el lenguaje, la cultura y las otras identidades (de género, raza o clase) que atraviesan a los sujetos (Nelson 1999, 377). De repente, todas las identidades sexuales se cuestionan y problematizan.

De cara a las repercusiones educativas, Nelson afirma que este enfoque, más abierto y flexible, proporciona al profesorado ventajas, puesto que no se espera que tengan todas las respuestas, sino que faciliten contextos de cuestionamiento, investigación y discusión $(1999,377)$.

Este giro copernicano en la pedagogía viene dado por la sacudida en la concepción de la identidad que supone la aparición de la teoría queer. La teoría queer (acuñada en 1991 por de Lauretis) es un conjunto de discursos y prácticas críticas que cuestionan el propósito y el funcionamiento de las identidades. Surge en Estados Unidos a mediados de los años1980, influenciada por el feminismo y la filosofía postestructuralista de Foucault y Derrida (Guijarro Ojeda 2006, 54).

El término queer (raro, extraño, desviado) nace como insulto a personas homosexuales, para luego ser reapropiado por los sujetos queer como motor de empoderamiento político. Aglutina lo abyecto, las identidades sexuales no normativas, anormales, y es una crítica a la victimización, a la normalización (Pié 2009, 253). La teoría queer entiende las identidades como actos culturales y discursivos, y considera que estas no son absolutas, inmutables, estáticas ni dicotómicas, sino fluidas.

Se prefiere traducir así el término pedagogies of inquiry, aunque Guijarro Ojeda (2006) opte por pedagogías de la investigación. 


\section{Pedagogía queer}

Lo que Britzman (1995) denomina pedagogía del cuestionamiento se ha venido articulando en los últimos años con el nombre de pedagogía queer, especialmente en Estados Unidos, Canadá y Australia. Está influida tanto por la pedagogía de la liberación de Paulo Freire, como por la pedagogía feminista y la crítica.

Dicen Zafra y Platero (Escuela de Educación Disruptiva 2015) que la pedagogía queer es una pedagogía trasformadora que persigue no solo evitar la discriminación, sino incorporar la otredad y subvertir las estructuras de opresión y normalidad, dentro de un esquema que pone hincapié en los procesos por encima del producto. Del Castillo (2015) comenta que es el modelo adecuado para prevenir y desmantelar la hetenormatividad en las aulas, restaurando el objetivo de la educación como práctica liberadora, para conseguir una sociedad más justa y equitativa.

No obstante, hay que aclarar que su misión no se limita a temas de género y sexualidad, sino a desestabilizar el binomio normal-anormal (Planella y Pié 2012), problematizar lo que es dado por hecho en la sociedad y promover la autorreflexión y la conciencia crítica. Estas dinámicas permiten a los docentes cuestionar su rol como educadores/as dentro del binomio profesor/a-alumno/a. En ese sentido, «el rol del educador cambia de una autoridad que sabe todo a la de un educador-alumno y facilitador»(del Castillo 2015).

\section{Otredad e interculturalidad en la enseñanza de lenguas}

La otredad es el «sentimiento de extrañeza que surge cuando el ser humano se enfrenta a todo aquello que le es desconocido, resultando a menudo en el rechazo causado por el miedo a lo alieno» (Flores 1999, 1, apud Guijarro Ojeda 2004b, 85). No obstante, es también a través de la otredad desde donde se configura la propia identidad, estableciendo los límites entre el yo y lo diferente en cuanto a género, sexualidad, raza, cultura, clase, etc. (Guijarro Ojeda 2005c, 65).

La otredad empieza a ser relevante para la enseñanza de lenguas extranjeras desde que, con el paradigma comunicativo, se privilegia la visión de la lengua como instrumento de comunicación, que permite intercambios entre personas de sociedades y culturas diferentes (Guijarro Ojeda 2005c, 66). Es también el momento en que la enseñanza de la lengua se concibe indisociable de su cultura.

En este contexto gana peso el concepto de interculturalidad, entendida como las relaciones que se establecen entre personas de diferentes culturas, partiendo del interés, el respeto y el reconocimiento mutuo de sus idiosincrasias. Paralelamente, también alude a la relación entre personas de una misma comunidad que pertenecen a diferentes grupos (de género, sexualidad, raza, cultura, clase, profesión, etc.) (Martín Peris 2008).

El enfoque intercultural constituye un elemento relevante en el aula de lengua extranjera puesto que permite al alumnado no solo reflexionar desde sí mismo y desde su propia identidad sino también tener una visión positiva de la 
diferencia como algo enriquecedor para su formación como individuo (Álvarez González 2010, 57).

Este enfoque intercultural se vuelca en los documentos curriculares actuales. No hay más que consultar el Plan curricular del Instituto Cervantes (Instituto Cervantes 2006) para apreciar la importancia que se le concede en su inventario de objetivos dedicados a la dimensión del alumno/a como hablante intercultural, pero también en Saberes y comportamientos socioculturales, Habilidades y actitudes interculturales, y Procedimientos de aprendizaje.

El currículo de ELE está atravesado por la interculturalidad, donde se promueve una superación de las posturas etnocéntricas y se potencia el respeto, la aceptación y la valoración del otro, desde sus diferencias y su individualidad (aunando lo común y lo diferente). En esta línea, es posible establecer un paralelismo con el ámbito de las sexualidades disidentes (donde lo LGBT es lo otro), pues la sexualidad constituye también un eje de identidad en los individuos, y también es parte de sus peculiaridades específicas. En conclusión, si el currículo se esfuerza en desterrar estereotipos, prejuicios y discriminación culturales, cabe entender como parte de este marco también la sexualidad.

\section{Factores afectivos}

Define Arnold (2006) la afectividad como el conjunto de aspectos relacionados con sentimientos, emociones, creencias, actitudes que influyen en nuestro comportamiento. Desde los años 1960 se ha venido estudiando su impacto en los procesos de aprendizaje-enseñanza, para concluir que la eficacia del aprendizaje está relacionada con la dimensión afectiva, pues esta es completamente indisociable, incluso a nivel neurobiológico, de lo cognitivo.

De hecho, incluso el Marco Común Europeo de Referencia del Consejo de Europa (MECD 2002) subraya la importancia de este plano en su apartado dedicado a la competencia existencial o saber ser (5.1.3.):

La actividad comunicativa de los usuarios o alumnos no sólo se ve afectada por sus conocimientos, su comprensión y sus destrezas, sino también por factores individuales relacionados con su personalidad y caracterizados por las actitudes, las motivaciones, los valores, las creencias, los estilos cognitivos y los tipos de personalidad que contribuyen a su identidad personal. (MECD 2002, 103)

En este paradigma educativo, el rol del profesorado adquiere vital trascendencia, pues el o la docente se erige como facilitador o facilitadora de los procesos de aprendizaje, que debe promover un clima relajado, de apoyo, valoración y respeto, y ayudar a resolver las posibles situaciones inhibidoras, conflictivas o de tensión.

En cuanto a la relevancia de la afectividad para el alumnado LGBT, hay que reconocer que se trata de un aspecto delicado, al encontrarse en el aula en una 
posición de vulnerabilidad. Esto sucede, por un lado, a causa de la invisibilización y ausencia de reconocimiento en los materiales. Asimismo, está relacionado con la falta de libertad para expresar libremente su orientación sexual, ante el peligro de exponerse a la estigmatización, discriminación o rechazo directos. Por eso, corren el riesgo de sufrir niveles más altos de ansiedad y baja autoestima.

Esta situación, en un contexto pedagógico de aprendizaje centrado en el alumno o alumna, no deja de ser perjudicial: «los estudiantes cuyas voces no están siendo reconocidas en el aula pueden perder su deseo de aprender el idioma o incluso desarrollar una resistencia pasiva a las prácticas de clase y las demandas curriculares» (Pavlenko 2004, 59, apud Pawelczyk et al. 2013).

Para subvertir esta dinámica, se propone más presencia y visibilidad en los materiales. Asimismo, hay que promover un ambiente que celebre las diferencias y respete las opiniones y realidades divergentes (El-Metoui 2014); un clima de empatía, donde se aprecie y reconozca la identidad del otro y se asuma que la forma de ser de uno no es la única ni quizá la mejor opción, lo que contribuye a una coexistencia armónica (Arnold y Brown 2000, 36).

\section{Metodología de la investigación}

El objetivo de la investigación es analizar la representación de la sexualidad en los manuales de ELE y, sobre todo, poner de manifiesto el papel que desempeñan estos en la transmisión y perpetuación de estereotipos y valores heterosexistas y LGBTfóbicos.

La elección de esta metodología no es baladí. Por un lado, los manuales son los materiales nucleares en la enseñanza del español, el input al que están expuestos miles de estudiantes. Asimismo, son instrumentos ideológicos, que reproducen los valores establecidos en la sociedad de la lengua meta, a través tanto de su plasmación explícita como de la omisión de ciertos elementos (el llamado currículo oculto): no existe aquello que no se nombra (Guijarro Ojeda 2005a, 156). Finalmente, son exponentes socioculturales representativos de la sociedad que los emite. $^{4}$

Se ha optado por un análisis cualitativo, ya que, como afirma Blat Gimeno, «la revisión de los textos no debe reducirse a la mera cuantificación del número de estereotipos, sino incluir también los aspectos ideológicos, lo que es más complejo y requiere la evaluación del significado de los mensajes ocultos» (1994, apud Lopo 2015, 57). Asimismo, este tipo de análisis resulta pertinente teniendo en cuenta la escasez de referencias a sexualidades no normativas encontradas.

El corpus se conforma de catorce manuales de ELE, cuya selección ha tenido en cuenta los siguientes aspectos:

A esto hay que añadir la cuestión aún sin resolver sobre la sociedad que deben reflejar los contenidos de los libros: ¿la sociedad tal como es o como deseamos que fuera? 
- Forman parte de colecciones para enseñanza a jóvenes y adultos.

- Pertenecen a diferentes editoriales españolas de reconocido multinivel para prestigio y presencia real en el mercado editorial.

- Son posteriores a 2006, publicados después de dos hitos legislativos y curriculares: por un lado, la aprobación del matrimonio entre personas del mismo género (Ley 13/2005, reforma del Código civil que posibilita el matrimonio entre personas del mismo sexo en España) y el aumento de la visibilidad mediática de la comunidad LGBT; por otro lado, la publicación del MCER (MECD 2002) y el PCIC (Instituto Cervantes 2006), con su atención a la otredad y al respeto de las identidades personales.

- Son representativos de las tendencias metodológicas de la actualidad.

- Se manejan únicamente los libros del alumno o alumna, prescindiendo de materiales complementarios.

- Son ediciones españolas estándar (aunque con la consciencia de que existen versiones adaptadas a diferentes contextos culturales).

Atendiendo a estos criterios, el corpus se compone de las siguientes obras:

- Editorial Difusión: Aula 1, 2, 3, 4, 5, 6 (Corpas, García, y Garmendia 2013; Corpas, Garmendia, y Soriano 2013a, 2013b, 2013c; Corpas et al. 2014a, 2014b).

- Editorial Edelsa: Pasaporte ELE A1, A2, B1 (Cerrolaza, Cerrolaza y Llovet 2007, 2008, 2009).

- Editorial Edinumen: Nuevo Prisma A1, A2 (Equipo nuevo Prisma 2012, 2013).

- Editorial enClave-ELE: En acción 2, 3, 4 (Verdía et al. 2011, 2010; Gutiérrez, Blas, y Abia 2010).

En cuanto a la naturaleza del análisis, cabe apuntar que se fija en la información textual ${ }^{5}$ (input lingüístico). Así, se tienen en cuenta tanto textos explicativos como enunciados y actividades (de todos los temas y dinámicas), en las menciones explícitas e implícitas que realicen a cuestiones de sexoafectividad.

\section{Análisis e interpretación crítica de los datos obtenidos}

A partir de la lectura del corpus, han podido establecerse cuatro mecanismos para incorporar la sexualidad a los manuales: mediante la caracterización de personajes, como tema, a través de las exhortaciones al alumnado y con referentes socioculturales. 
En primer lugar, respecto a los personajes, cabe subrayar que los manuales son polifónicos, en el sentido de que aparecen muchos personajes y voces en ellos. Estas voces (que pueden ser anónimas o presentarse con nombre) se utilizan tanto como hilo vertebrador de secuencias didácticas, o como simples exponentes lingüísticos para practicar objetivos y contenidos diversos. A veces, dichos personajes tienen cierta relevancia y recurrencia, y se aportan datos sobre su vida y carácter; en cambio, muchas otras, simplemente aparecen en una frase de un ejercicio y no vuelven a utilizarse. Además, en algunos casos, no es posible determinar su género; por lo tanto, su sexualidad queda en el aire (si bien la heteronormatividad permea su lectura heterosexual). Algo parecido ocurre cuando se emplean palabras como pareja, amor o cónyuge para hablar de los/as compañeros/as sentimentales: de nuevo tiende a interpretarse en clave heterosexual. En otros ejemplos, sí es evidente el sexo de ambos integrantes de una relación: siempre son heterosexuales. No hay en este punto ningún ejemplo que de manera explícita aluda a una relación homosexual, lésbica o bisexual. Esto evidencia lo anticipado en el apartado teórico: el currículo de ELE es heterosexista.

- Ítem 1 (Nuevo Prisma A1): A través de una pareja heterosexual, Nieves y Javi, se introduce y practica el léxico de la casa y los marcadores espaciales (Equipo nuevo Prisma 2012, 30).

- Ítem 2 (Aula 2): En este ejercicio de contraste entre ser y estar se incluye la siguiente frase: «Mi marido es alemán, de Dortmund». Esta vez no se especifica el sexo de la persona que emite el mensaje, por lo que no puede asegurarse si se encuadra en una relación heterosexual, aunque sea la lectura imperante (Corpas, Garmendia, y Soriano 2013a, 170).

- Ítem 3 (Pasaporte ELE A1): Incluso en un ejercicio de prosodia aparece la (hetero)sexualidad: "¿Juan no tiene novia?, Juan no tiene novia, ¡Juan no tiene novia!» (Cerrolaza, Cerrolaza y Llovet 2007, 36).

- Por otro lado, en cuanto a su desarrollo como tema, la sexualidad es el eje de contenidos sobre la familia, los estados civiles, las relaciones afectivas o las celebraciones. Los ejemplos tienden a cumplir los patrones heterosexuales y tradicionales. Siguiendo el planteamiento de Guijarro Ojeda (2005a, 164), no se está aprovechando el potencial que poseen estos temas, pues «junto a la familia podrían explotarse temas de orientación sexual, de género, de roles sociales, de identidad, de sexualidad, de educación, etc.»

- Ítem 4 (Pasaporte ELE A1): El manual introduce el vocabulario de la familia a través de la familia real española, cuya referencia, si bien rica para el acervo cultural hispánico, no es la más cercana ni representativa de la realidad española o la del alumno o alumna. Una vez introducido el léxico, se traslada la acción al discente, para que realice su propio árbol genealógico. La plantilla que se le facilita para tal tarea es únicamente heteronormativa (abuelo-abuela, madre-padre), lo que coarta la libertad del alumno 
o alumna en caso de que su familia no se corresponda con dicho modelo (Cerrolaza, Cerrolaza y Llovet 2007, 34).

- Ítem 5 (Pasaporte ELE A1): Una actividad muy recurrente también es la de buscar pareja. En este caso, el objetivo es emparejar a tres mujeres con tres hombres según su compatibilidad de caracteres. Las parejas hombre-mujer parecen ser la única combinación posible, ya que incluso se preparan las respuestas con la estructura él y ella (Cerrolaza, Cerrolaza y Llovet 2007, 42). - Volviendo al tema de la familia, es precisamente este un emplazamiento recurrente para las menciones a lo LGBT. En caso de aparecer una referencia, suele presentarse dentro de apartados que hablan de familias alternativas o nuevos tipos de familia, nunca en el modelo de familia que introduce el tema. El otro encuadre habitual para las sexualidades disidentes es en temas de debate o discusión sobre los cambios en la sociedad. Teóricamente, esto se relaciona con el concepto de otredad y la concepción de lo diferente como amenazador o conflictivo.

- Ítem 6 (Aula 2): Este manual incorpora pareja homosexual dentro de los nuevos tipos de familia, al mismo nivel que familia monoparental, parejas de hecho, familias con hijos adultos y personas mayores a su cargo, familias con hijos adoptivos y personas mayores de 35 años que comparten piso. También se enuncia el siguiente dato sociocultural, para que los alumnos y alumnas reflexionen sobre si existen situaciones parecidas o diferentes en sus respectivos países de origen: «22 442 bodas entre personas del mismo sexo de 2005 a 2012» (Corpas, Garmendia, y Soriano 2013a, 44).

- Ítem 7 (Pasaporte ELE B1): La primera unidad del manual trabaja sobre la juventud española, sus características y preocupaciones. Precisamente, sobre este último punto se presenta un texto que alude a la homosexualidad: «La solidaridad y la justicia son las causas sociales por las que las personas jóvenes se esfuerzan. Las actitudes tolerantes sobre cuestiones como la homosexualidad o la eutanasia contrastan con la firmeza contra conductas que ponen en riesgo el buen funcionamiento social». Es positivo que se incluya la homosexualidad en una unidad sobre la juventud, pero es cuestionable la manera en que se hace: equiparándola en tolerabilidad a la eutanasia, y presentada como algo extraño o de conflicto social más que como natural y normalizado (Cerrolaza, Cerrolaza y Llovet 2009, 8).

- Ítem 8 (En acción 3): Se utiliza una noticia sobre la aprobación parlamentaria del matrimonio entre parejas del mismo género para trabajar las funciones referentes a valorar hechos $\mathrm{u}$ opiniones. La actividad se articula en torno a la conversación entre dos personajes, Casto y Libertad, que comentan dos temas de actualidad (la aprobación del matrimonio entre personas del mismo sexo y un estudio sobre la posibilidad de prohibir fumar en la calle). Cada uno de ellos defiende una postura utilizando los exponentes lingüísticos de valoración. Se incorpora lo LGBT, pero nuevamente 
como fuente de discusión o polémica. Luego los o las estudiantes pueden reflexionar sobre el tema y compartir sus opiniones con sus compañeros (Verdía et al. 2010, 17).

- En tercer lugar, la sexualidad se infiltra en las exhortaciones al alumnado para que comparta sus experiencias vitales. En ocasiones, se le pide al discente participar relatando cuestiones de su vida que atañen a la sexualidad. Se le pregunta de manera desdoblada (sobre su novio/novia) o neutra (pareja). No obstante, el desdoblamiento no remite a un enfoque de diversidad sexual, sino que se presupone la heterosexualidad pero se dobla para englobar a alumnos y alumnas. Este tipo de mención se relaciona estrechamente con lo visto sobre los factores afectivos en el apartado de fundamentación teórica.

- Ítem 9 (Aula 1): En un ejercicio para practicar el pretérito perfecto, los/ as alumnos/as tienen que responder si han hecho algunas de las siguientes actividades: casarse, enamorarse, etc. Al hablar de las relaciones afectivas, pueden salir a la luz sexualidades disidentes, con el correspondiente estrés o ansiedad para el alumno o alumna, expectante de si experimentará rechazo o discriminación de sus compañeros y compañeras (Corpas, García, y Garmendia 2013, 170).

- Ítem 10 (En acción 3): Se insta a los/as alumnos/as a que piensen cosas de su vida (personas importantes, hechos o experiencias, fechas, etc.) que sus compañeros/as no saben de ellos, para luego contárselas. Paralelamente, se exhorta a los/as alumnos/as a preparar preguntas para su profesor/a, que remitan a curiosidades sobre la vida de este que quieran conocer. En esta actividad de cohesión grupal pueden aflorar experiencias asociadas a la sexualidad, tanto desde la perspectiva del docente como la de los/as alumnos/as, lo que puede causar estrés en alumnos/as y docentes LGBT (Verdía et al. 2010, 14).

- Por último, cabe apuntar la cantidad de iconos socioculturales que incorporan la sexualidad, tanto en forma de personajes de la escena social y cultural hispana, como a través de obras representativas de la cultura.

- Ítem 11 (Nuevo Prisma A1): Es habitual en los manuales que se mencione la heterosexualidad de iconos de la cultura hispana (se hallan ejemplos de Miguel de Cervantes, Isabel Allende, Penélope Cruz, Michelle Bachelet, Evita Perón, Vicente Ferrer, Icíar Bollaín, etc.). En el caso de Penélope Cruz, se alude a que «está casada con el actor Javier Bardem y tienen un hijo que se llama Leo» (Equipo nuevo Prisma 2012, 42). No obstante, cuando se trata de personajes que no son heterosexuales (Federico García Lorca, Reinaldo Arenas, Frida Kahlo, Pedro Almodóvar, Victorio \& Lucchino o Jaime Gil de Biedma) se omite cualquier referencia a su orientación sexual.

- Como ya se explicó en el marco teórico, esta invisibilización acarrea consecuencias para el aprendizaje de los/as estudiantes LGBT. Esta invisibili- 
zación se relaciona con el hecho de que las identidades LGBT se conciban hipersexualizadas, cosa que no ocurre con la heterosexualidad.

- Ítem 12 (Nuevo Prisma A1): Esta actividad (Equipo nuevo Prisma 2012, 34) hace hincapié en el léxico del barrio, al tiempo que introduce contenidos culturales e interculturales sobre los diferentes vecindarios de Madrid. A través del personaje de Almudena se presenta el barrio de Chueca; no obstante, solo se comenta: «En el barrio hay un ambiente muy intercultural porque se mezclan personas de diferentes provincias». Es significativo que no se mencione en ningún momento a la comunidad LGBT, teniendo en cuenta que Chueca es un epicentro de la diversidad sexual no solo en Madrid, sino en España y Europa. Por el contrario, en En acción 4 (Gutiérrez, Blas, y Abia 2010, 79), en una actividad sobre propuestas culturales en Madrid, se habla de Chueca como «el centro de la vida gay de Madrid y de España».

Tras la observación y examen del corpus, se puede concluir que las sexualidades no normativas están sumamente infrarrepresentadas. En un corpus de más de mil páginas, se constata que las referencias directas no superan la docena. En contraposición, los manuales están repletos de referencias explícitas e implícitas a la heterosexualidad, no solo en las unidades que desarrollan temas como la familia, los estados civiles o las relaciones afectivas, sino atravesando los diferentes objetivos y contenidos de los libros.

En concreto, el método Nuevo Prisma (A1 y A2) no recoge ninguna mención explícita a la diversidad sexoafectiva (Equipo nuevo Prisma 2012, 2013). En la colección Pasaporte ELE (A1, A2, B1) aparecen dos alusiones a la homosexualidad, entendida como foco de debate (Cerrolaza, Cerrolaza y Llovet 2007, 2008, 2009). En acción (2, 3 y 4; esto es, niveles B1, B2 y C1) es la colección que incorpora más veces las sexualidades disidentes (un total de cinco), algunas incluso como parte de los objetivos y contenidos del manual (Verdía et al. 2011, 2010; Gutiérrez, Blas, y Abia 2010). No obstante, la desproporción con respecto a la heterosexualidad continúa siendo evidente. Por último, la colección Aula incluye otras dos referencias a la homosexualidad, presentada dentro del espectro de la diversidad familiar (Corpas, García, y Garmendia 2013; Corpas, Garmendia, y Soriano 2013a, 2013b, 2013c; Corpas et al. 2014a, 2014b).

Finalmente, es relevante apuntar un par de tendencias generales halladas en el corpus.

Las menciones LGBT encontradas no pertenecen a niveles iniciales, sino intermedios o avanzados, lo que concuerda con los datos hallados en el PCIC. En dicho documento, la diversidad sexual está presente en las nociones específicas del nivel B1. Cabe preguntarse esta posición relegada en el currículum, que deja entrever que la orientación sexual se interpreta como un rasgo secundario, en comparación con otros aspectos de la dimensión física, anímica o relacional del individuo. 
Por otro lado, todas las menciones a sexoafectividades fuera de la norma remiten exclusivamente a la homosexualidad, lo que invisibiliza identidades específicas (gais, lesbianas) o identidades no homosexuales (bisexualidad y otras identidades plurisexuales).

\section{Conclusiones}

Como se ha podido contrastar a través del análisis del corpus, las sexualidades no normativas son marginales y están (prácticamente) silenciadas y discriminadas en los manuales de ELE, que reproducen un régimen de heteronormatividad. Esta situación es, además de injusta, contraproducente para toda la comunidad educativa (no solamente estudiantes y docentes LGBT), pues puede acarrear consecuencias para el aprendizaje.

Este artículo se convierte en una llamada a la responsabilidad, tanto de editoriales, como de docentes y programas de formación de profesorado, para revertir este panorama. Ahora bien, la solución no pasa por incluir de manera esporádica y superficial personajes LGBT en el currículo (pedagogía de la inclusión), sino por cuestionar los privilegios y tomar consciencia de las opresiones que atraviesan las identidades sexuales y de género (pedagogía queer). El objetivo no es conseguir aulas más tolerantes, sino más libres y críticas. En definitiva, se trata de celebrar las diferencias, y combatir las desigualdades y discriminaciones.

\section{Referencias bibliográficas}

Álvarez González, Severina. 2010. «La relevancia del enfoque intercultural en el aula de lengua extranjera». Revista Nebrija de Lingüística Aplicada 9: 40-58.

Arnold, Jane. 2006. «Los factores afectivos en el aprendizaje del español como lengua extranjera». Disponible en http://cvc.cervantes.es/ensenanza/biblioteca_ele/antologia_ didactica/claves/arnold.htm. [Consultado el 14 de mayo de 2017].

Arnold, Jane, y Douglas Brown. 2000. «Mapa del terreno». En La dimensión afectiva en el aprendizaje de idiomas, editado por Jane Arnold, 19-36. Madrid: Cambridge University Press.

Britzman, Deborah. 1995. «Is There A Queer Pedagogy? Or, Stop Reading Straight». Educational Theory 45 (2): 151-165. DOI: https://doi.org/10.1111/j.1741-5446.1995.00151.x

Cartwright, Ivan. 2006. «Mind the gap: an investigation into perceived diversity gap in the English language learning sub-culture». Language Issues, 18 (2): 176-183.

Cerrolaza, Matilde, Óscar Cerrolaza, y Begoña Llovet. 2007. Pasaporte ELE A1. Madrid: Edelsa.

Cerrolaza, Matilde, Óscar Cerrolaza, y Begoña Llovet. 2008. Pasaporte ELE A2. Madrid: Edelsa.

Cerrolaza, Matilde, Óscar Cerrolaza, y Begoña Llovet. 2009. Pasaporte ELE B1. Madrid: Edelsa.

Corpas, Jaime, Eva García, y Agustín Garmendia. 2013. Aula 1. Barcelona: Difusión. 
Corpas, Jaime, Agustín Garmendia, y Carmen Soriano. 2013a. Aula 2. Barcelona: Difusión. Corpas, Jaime, Agustín Garmendia, y Carmen Soriano. 2013b. Aula 3. Barcelona: Difusión. Corpas, Jaime, Agustín Garmendia, y Carmen Soriano. 2013c. Aula 4. Barcelona: Difusión.

Corpas, Jaime, Agustín Garmendia, Nuria Sanchez, y Carmen Soriano. 2014a. Aula 5. Barcelona: Difusión.

Corpas, Jaime, Agustín Garmendia, Nuria Sanchez, y Carmen Soriano. 2014b. Aula 6. Barcelona: Difusión.

del Castillo, Valeria. 2015. «Heteronormativity and Classroom Strategies: An Educator's Guide to Queering the Normal». Disponible en https://www.academia.edu/6035720/ Heteronormativity_and_Classroom_Strategies. [Consultado el 14 de mayo de 2017].

El-Metoui, Laila. 2012. «Breaking the Ice. Fostering a positive atmosphere inclusive of LGBT issues in the ESOL Classroom». Disponible en https://nateclaldn.files.wordpress. com/2012/10/breaking-the-ice-pptx.pdf. [Consultado el 14 de mayo de 2017].

El-Metoui, Laila. 2014. «LGBT issues in English teaching in the UK», en Voices Magazine. Disponible en http://www.britishcouncil.org/voices-magazine/english-language-teachers-address-lgbt-issues-class, consultado el 14 de mayo de 2017.

Equipo nuevo Prisma. 2012. Nuevo Prisma, nivel A1. Madrid: Edinumen.

Equipo nuevo Prisma. 2013. Nuevo Prisma, nivel A2. Madrid: Edinumen.

Erlman, Lisa. 2015. «Heteronormativity in EFL textbooks. A review of the current state of research on gender-bias and heterosexism in ELT reading material». Disponible en https://gupea.ub.gu.se/bitstream/2077/38648/1/gupea_2077_38648_1.pdf. [Consultado el 14 de mayo de 2017].

Escuela de Educación Disruptiva. 2015. «Salir del armario: pedagogías queer y otras formas de abordar la identidad». Disponible en https://www.youtube.com/watch?v=h9hYQMNWuzU. [Consultado el 14 de mayo de 2017].

Gray, John. 2013. «LGBT invisibility and heteronormativity in ELT materials». Critical Perspectives on Language Teaching Materials, editado por John Gray, 40-63. Basingstoke: Palgrave Macmillan.

Guijarro Ojeda, Juan Ramón. 2004a. «Addressing queer/GLTB issues in the EFL classroom within the Spanish educational context». The Grove: Working Papers On English Studies 4: 59-70.

Guijarro Ojeda, Juan Ramón. 2004b. «Cine y otredad en ELT: educar a través de las voces silenciadas de Billy Elliot». Odisea 5: 81-94.

Guijarro Ojeda, Juan Ramón. 2005a. «La representación axiológica del género y la orientación en libros de texto de inglés para secundaria». Porta Linguarum 4: 151-166.

Guijarro Ojeda, Juan Ramón. 2005b. «Valores de otredad (cultura y género) en los libros de texto de inglés para primaria». Encuentro: revista de investigación e innovación en la clase de idiomas 15: 32-38.

Guijarro Ojeda, Juan Ramón. 2005c. «Conceptualización e implicaciones didácticas de la otredad para el área de inglés como lengua extranjera (EFL)». Miscelánea: A Journal of English and American Studies 31: 65-88.

Guijarro Ojeda, Juan Ramón. 2006. «Enseñanzas de la teoría Queer para la didáctica de la lengua y la literatura extranjeras». Porta Linguarum 6: 53-66.

Guijarro Ojeda, Juan Ramón, y Raúl Ruiz-Cecilia. 2013. «Perceptions of Spanish EFL trainee teachers on the introduction of queer issues in the classroom». Onomázein: revista de lingüística, filología y traducción de la Pontificia Universidad Católica de Chile 27: 193-206.

Gutiérrez, Esther, Amelia Blas, y Belén Abia. 2010. En acción 4, Madrid: EnClave-ELE. 
Instituto Cervantes. 2006. Plan curricular del Instituto Cervantes. Niveles de referencia para el español. Madrid: Instituto Cervantes: Biblioteca Nueva. Disponible en http://cvc.cervantes.es/ensenanza/biblioteca_ele/plan_curricular/. [Consultado el 14 de mayo de 2017].

Ley 13/2005, de 1 de julio, "por la que se modifica el Código Civil en materia de derecho a contraer matrimonio». En BOE, 157, 2 de julio de 2005, 23632-23634. Disponible en https://www.boe.es/buscar/doc.php?id=BOE-A-2005-11364. [Consultado el 14 de mayo de 2017].

Lluch, Antoni. 2010. «¿Sexualidad en la clase de ELE? Sí, por favor». En Actas del XVIII Seminario de Dificultades de la Enseñanza del Español a Lusohablantes, 53-74. São Paulo: Embajada de España en Brasil y Consejería de Educación.

Lopo, María. 2015. «Sexismo y coeducación: la discriminación genérica en los manuales de lengua francesa». Encuentro 15: 55-65.

Macdonald, Sheila. 2014. «Out in the classroom?: Exploring LGBT lives and issues in adult ESOL». En Language issues in migration and integration: perspectives from teachers and learners, editado por David Mallows, 153-169. London: British Council.

Martín Peris, Ernesto. 2008. Diccionario de términos clave de ELE. Madrid: SGEL.

Mazzaro, Daniel. 2013. «iNo a la homofobia! Contribuciones del profesor de español a la enseñanza sin homofobia». En Anais XIV Congresso Brasileiro de Professores de Espanhol, 381-394. Rio de Janeiro: APEERJ.

Mazzaro, Daniel, y Luiza Chaves. 2010. «Hablando del respeto a las diferencias y de la sexualidad en las clases de español en la secundaria». En Actas del XVIII Seminario de Dificultades de la Enseñanza del Español a Lusohablantes, 119-134. São Paulo: Embajada de España en Brasil y Consejería de Educación.

MECD - Ministerio de Educación, Cultura y Deporte. 2002. Marco común europeo de referencia para las lenguas: aprendizaje, enseñanza, evaluación. Madrid: MECD y Anaya. Disponible en https://cvc.cervantes.es/ensenanza/biblioteca_ele/marco/cvc_mer.pdf

Nelson, Cynthia. 1993. «Heterosexism in ESL: Examining our attitudes». TESOL Quarterly 27 (1): 143-150. DOI: https://doi.org/10.2307/3586966

Nelson, Cynthia. 1999. "Sexual identities in ESL: Queer Theory and classroom inquiry». TESOL Quarterly 33 (3): 371-392. DOI: https://doi.org/10.2307/3587670

Pawelczyk, Joanna, Lukasz Pakuła, y Jane Sunderland. 2013. «Investigating gender and sexuality in the ESL classroom: Raising publishers', teachers' and students' awareness». Disponible en http://www.academia.edu/6233574/Investigating_Gender_and_Sexuality_in_the_ESL_classroom_Raising_publishers_teachers_and_students_awareness_--_a_promo_brochure. [Consultado el 14 de mayo de 2017].

Pié, Asun. 2009. «De la teoria queer i les altres maneres de pensar l'educació». Temps d'Educació 37: 253-270.

Planella, Josep, y Asun Pié. 2012. «Pedagoqueer: resistencias y subversiones educativas». Educación XX115: 265-283. DOI: https://doi.org/10.5944/educxx1.15.1.159

Summerhawk, Barbara. 1998. «From closet to classroom: Gay issues in ESL/EFL». The Language Teacher (Japan Association for Language Teaching) 22 (5): 21-23.

Verdía, Elena et al. 2010. En acción 3. Madrid: EnClave-ELE.

Verdía, Elena et al. 2011. En acción 2. Madrid: EnClave-ELE.

Warner, Michael. 2011. «Introduction: Fear of a Queer Planet». Social Text, 29: 3-17. 

EXTRANJERA

Maria Salvia Rodríguez. Nacida en Barcelona (1988), es licenciada en Filología Hispánica (2010) por la Universitat de Barcelona, máster en Léxico y comunicación lingüística (2011) por la misma universidad, en Dirección de comunicación empresarial e institucional (2014) por la Universitat Autònoma de Barcelona, y en Enseñanza del español como lengua extranjera (2015) por la Universidad Internacional Menéndez Pelayo. Su investigación se centra en la interacción entre lingüística, didáctica de lenguas y estudios de género. Correo electrónico: msalviarod@gmail.com

Artículo recibido el 14 de mayo de 2017 y aceptado para su publicación el 28 de septiembre de 2017. 
